SELECCIONES MATEMÁTICAS
Universidad Nacional de Trujillo
ISSN: $2411-1783$ (Online)
Vol. $05(02): 241-248(2018)$

\title{
Sobre el Espacio Euclidiano Bidimensional
}

\section{On the Bidimensional Euclidean Space}

Edgar Vera Saravia* ${ }_{\text {and }}$ Henry Zubieta Rojas ${ }^{\dagger}$

Received, Jun. 12, 2018

Accepted, Nov. 10, 2018

DOI: http://dx.doi.org/10.17268/sel.mat.2018.02.10

\begin{abstract}
Resumen
Ofrecemos una introducción geométrica del Espacio Euclideano Bidimensional $\mathbb{E}^{2}$ utilizando, tanto el enfoque geométrico del $\mathbb{R}$-Espacio Afin Bidimensional como el Álgebra Geométrica Bidimensional.

Estas notas, dirigidas a Docentes Universitários de Física y Matemática, tratan de emular lo propuesto por Felix Klein en la primera década del siglo XX.

Palabras clave: Álgebra Geométrica, Espacio Euclideano, Espacio Afin.
\end{abstract}

\begin{abstract}
We offer a geometric introduction to the two-dimensional Euclidean Space $\mathbb{E}^{2}$ using both the geometric approach of the affine $\mathbb{R}$-Space and the two-dimensional Geometric Algebra.

These notes, intented for university proffessors of physics and mathematics, try to emulate the program developed by Felix Kline in the first decade of the 20th century.
\end{abstract}

Keywords: Geometric Algebra, Euclidean Space, Afin Space.

1. Introducción. En su Erlanger Programm de 1908 Felix Klein propuso:

Un docente puede abordar con mayor solvencia y convicción el tema de matemática que le corresponda transmitir a sus alumnos, estudiando previamente dicho tema desde un punto de vista matemático avanzado.

Klein plasmó sus ideas en su libro, para Docentes Preuniversitários de Matemática: Matemática Básica desde un punto de vista superior, acorde con la evolución matemática hasta el siglo XIX.

Pretendemos hacer Matemática Básica para Docentes Universitários de Física y Matemática,

- Mostrando el aspecto avanzado de la misma y

- Acompañando su evolución hasta el siglo XX.

En este contexto ofrecemos una versión matemática amigable de dos temas que enriquecen las demostraciones geométricas clásicas mediante demostraciones algebraicas de las mismas,

PRIMERO: El Plano Euclideano Bidimensional $\mathbb{E}^{2}$ que en las aplicaciones, sin mayor preámbulo, es representado mediante una pizarra, una hoja de papel o una pantalla, que denotaremos $\mathcal{P}$, utilizando

- Puntos $\in \mathcal{P}$ (denotados A, B, C), para representar los elementos de $\mathbb{E}^{2}$.

- Flechas $\subset \mathcal{P}$ (denotadas $\mathrm{AB}, \mathrm{BC}, \mathrm{AC}$ ) para representar los vectores libres de $\mathbb{E}^{2}$. Matematizaremos $\mathbb{E}^{2}$ considerando el conjunto de pares ordenados de números reales $\mathfrak{R}_{2} \equiv \mathbb{R} \times \mathbb{R}$ de tal modo que todo punto sirve de origen de una estructura de espacio vectorial y dichos espacios vectoriales resultan convenientemente conectados entre si y son isomorfos a $\mathbb{R}^{2}$ (el conjunto $\mathfrak{R}_{2}$ provisto de la estructura usual de espacio vectorial). Esto es parte de lo que se necesita para algebrizar las demostraciones geométricas en el espacio euclideano.

\footnotetext{
${ }^{*}$ Facultad de Ciencias Matemáticas - UNMSM, Av. Venezuela s.n., Lima-Perú (everas@unmsm. edu . pe).

${ }^{\dagger}$ Unidad de Posgrado - FCM - UNMSM, Av. Venezuela s.n., Lima-Perú (hzubietar@unmsm.edu.pe).
} 
SEGUNDO: El Álgebra Geométrica Bidimensional AG(2) que mejora el conocido encaje de álgebras determinado por los reales y complejos $(\mathbb{R}$ es subálgebra de $\mathbb{C})$ :

$$
\mathbb{R}<\mathbb{C}
$$

Como sabemos, los complejos ampliaron el análisis real e incluyeron, de modo indirecto, algunos aspectos geométricos como las rotaciones.

Presentaremos $\mathrm{AG}(2)$ como una abstracción geométrica del álgebra no conmutativa de matrices reales $\mathbb{R}^{2 \times 2}$ y obtendremos el siguiente encaje de álgebras reales:

$$
\mathbb{R}<\mathbb{C}<A G(2)
$$

2. Bosquejando el Espacio Euclideano Bidimensional. El enfoque geométrico de los espacios afines trata de un tipo de variedad $\mathcal{A}$ que tiene la propiedad de que tanto ella como su fibrado tangente $T \mathcal{A} \equiv \mathcal{A} \times \mathcal{A} \longrightarrow \mathcal{A}$ son representados en un mismo ambiente: Los elementos de $\mathcal{A}$ son representados por puntos y los elementos de $T \mathcal{A}$ por flechas (también llamadas vectores libres).

En este contexto el Espacio Euclideano Bidimensional $\mathbb{E}^{2}$ es matematizado como el conjunto de pares ordenados de números reales $\mathfrak{R}_{2} \equiv \mathbb{R} \times \mathbb{R}$ provisto de la siguiente estructura:

EA1. Con $\mathbb{R}^{2}$ denotaremos el conjunto $\mathfrak{R}_{2}$ provisto de la estructura usual de $\mathbb{R}$-espacio vectorial.

EA2. Todo punto sirve de origen de una estructura de $\mathbb{R}$-espacio vectorial isomorfa a $\mathbb{R}^{2}$.

EA3. Representaremos $\mathbb{E}^{2}$ del modo tradicional, usando una pizarra, una hoja de papel o una pantalla, que denotaremos $\mathcal{P}$, de tal modo que:

- Cada elemento $P \in \mathbb{E}^{2}$ es representado por un único punto $P \in \mathcal{P}$. Usaremos la misma letra $\mathrm{P}$ en ambos casos.

- Los elementos $(P, Q) \in \mathbb{E}^{2} \times \mathbb{E}^{2}$ son representados por flechas $P Q \subset \mathcal{P}$, con origen el punto asociado a $\mathrm{P}$ y extremo el punto asociado a $\mathrm{Q}$. También usaremos $\mathrm{PQ}$ para referirnos al par ordenado $(\mathrm{P}, \mathrm{Q})$ y en ambos casos será llamado vector tangente de $\mathbb{E}^{2}$ en $P \in \mathbb{E}^{2}$.

$T_{P} \mathbb{E}^{2}=\{P\} \times \mathbb{E}^{2}$ indicará la familia de los vectores tangentes de $\mathbb{E}^{2}$ en $P \in \mathbb{E}^{2}$, representados con flechas con origen en $P$.

EA4. Se establece una Conexión entre los diferentes espacios tangentes mediante la llamada Traslación Paralela que determina:

- Cada vector tangente $X Y \in T_{X} \mathbb{E}^{2}$ es biunívocamente asociado a un vector tangente $\underline{X Y}:=O Y-O X \in T_{O} \mathbb{E}^{2}$, donde $O=(0,0)$,

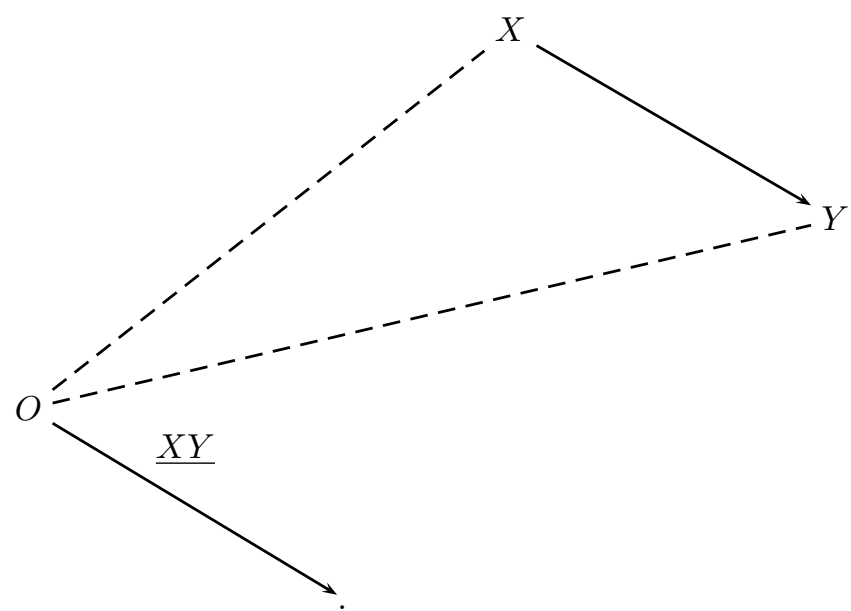

Resulta un isomorfismo, como $\mathbb{R}$-espacios vectoriales, $T_{X} \mathbb{E}^{2} \equiv T_{O} \mathbb{E}^{2} \equiv \mathbb{R}^{2}$, que permite

- Hablar de vectores libres paralelos,

$$
P X \in T_{P} \mathbb{E}^{2} \text { y } Q Y \in T_{Q} \mathbb{E}^{2} \text { son igualmente paralelos } \Longleftrightarrow \underline{P X}=\underline{Q Y}
$$

- Sumar vectores libres cualesquiera y obtener un vector en $T_{R} \mathbb{E}^{2}$, con $R \in \mathbb{E}^{2}$ prefijado, mediante la suma de vectores en $\mathbb{R}^{2}$ :

$$
P X+Q Y=R Z \Longleftrightarrow \underline{P X}+\underline{Q Y}=\underline{R Z}
$$


Con esto podemos matematizar la suma de vectores libres

$$
A B+B C=A C
$$

que tradicionalmente se "presenta y demuestra" recurriendo al "criterio del paralelogramo"

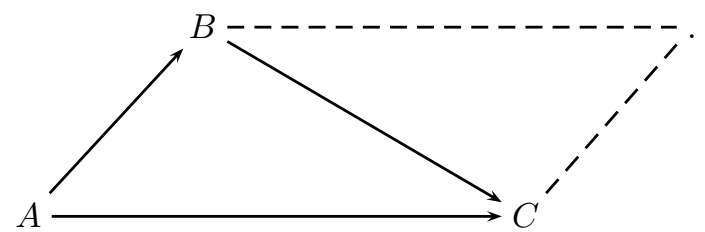

utilizando ahora

$$
A B+B C=A C \Longleftrightarrow \underline{A B}+\underline{B C}=\underline{A C}
$$

la última suma es en $\mathbb{R}^{2}$ y se representa con el paralelogramo

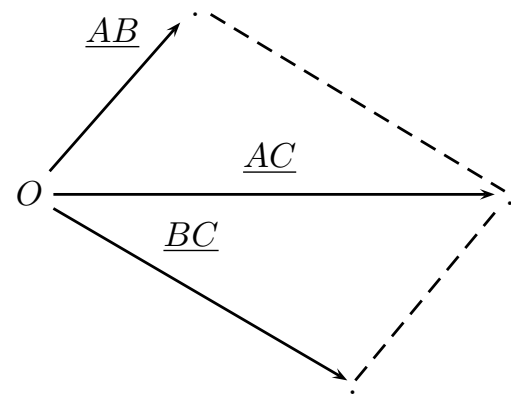

3. Bosquejando el Algebra Geométrica Bidimensional. Las matrices, pertenecientes al álgebra de matrices reales $\mathbb{R}^{2 \times 2}$,

$$
m_{0}=\left(\begin{array}{ll}
1 & 0 \\
0 & 1
\end{array}\right), \quad m_{1}=\left(\begin{array}{ll}
0 & 1 \\
1 & 0
\end{array}\right) \quad y \quad m_{2}=\left(\begin{array}{cc}
1 & 0 \\
0 & -1
\end{array}\right)
$$

tienen propiedades realmente maravillosas:

$$
\left\{m_{0}, m_{1}, m_{2}, m_{1} m_{2} \equiv m_{12}\right\}
$$

es una base de $\mathbb{R}^{2 \times 2}$, como $\mathbb{R}$-espacio vectorial; precisamente,

$$
\mathbb{R}^{2 \times 2}=\left\{a_{0}+a_{1} m_{1}+a_{2} m_{2}+a_{3} m_{12} ; a_{j} \in \mathbb{R} .\right\}
$$

Explicitamente los elementos de la base son:

$$
\left(\begin{array}{ll}
1 & 0 \\
0 & 1
\end{array}\right), \quad\left(\begin{array}{ll}
0 & 1 \\
1 & 0
\end{array}\right), \quad\left(\begin{array}{cc}
1 & 0 \\
0 & -1
\end{array}\right) \text { y }\left(\begin{array}{cc}
0 & -1 \\
1 & 0
\end{array}\right)
$$

Los productos entre estas matrices determinan la siguiente tabla multiplicativa

\begin{tabular}{c|c|c|c}
$m_{0}$ & $m_{1}$ & $m_{2}$ & $m_{12}$ \\
\hline$m_{1}$ & $m_{0}$ & $m_{12}$ & $m_{2}$ \\
\hline$m_{2}$ & $-m_{12}$ & $m_{0}$ & $-m_{1}$ \\
\hline$m_{12}$ & $-m_{2}$ & $m_{1}$ & $-m_{0}$
\end{tabular}

Esto sugiere la siguiente abstracción al "álgebra de matrices sin matrices” AG(2).

Definición 1. El Algebra Geométrica Euclideana Bidimensional AG(2) será el siguiente $\mathbb{R}$ - subespacio vectorial del anillo de polinomios $\mathbb{R}\left[u_{1}, u_{2}\right]$, en las variables $u_{1}$ y $u_{2}$ :

$$
A G(2):=\left\{a_{0}+a_{1} u_{1}+a_{2} u_{2}+a_{3} u_{1} u_{2} ; \quad a_{j} \in \mathbb{R}\right\}
$$


sus elementos serán llamados números geométricos.

$A G(2)$ estará provisto de un producto distributivo y asociativo, pero no conmutativo, llamado producto geométrico, procesado usando la siguiente tabla $\left(u_{1} u_{2} \equiv u_{12}\right)$

\begin{tabular}{c|c|c|c}
$l$ & $u_{1}$ & $u_{2}$ & $u_{12}$ \\
\hline$u_{1}$ & $l$ & $u_{12}$ & $u_{2}$ \\
\hline$u_{2}$ & $u_{21}$ & 1 & $-u_{1}$ \\
\hline$u_{12}$ & $-u_{2}$ & $u_{1}$ & -1
\end{tabular}

Tabla del producto geométrico de $A G(2)$

Una forma compacta de expresar la tabla del producto geométrico de AG(2) es mediante las llamadas Condiciones de Dirac:

$$
u_{i} u_{j}+u_{j} u_{i}=2 \delta_{i j} \quad \text { con } \quad i, j \in\{1,2\}
$$

Es pertinente precisar las operaciones de $\mathrm{AG}(2)$ : Su estructura vectorial nos permite sumar números geométricos pero, para multiplicar números geométricos debemos tener cuidado ya que, si bien se trata de un proceso similar al producto de polinomios en el sentido que solo podemos usar la distributividad y la asociatividad pero no la conmutatividad, porque el producto geométrico proviene del producto de matrices que no es conmutativo y en su lugar debemos utilizar la tabla (3.5). Veamos esto, dados

$$
A=a_{0}+a_{1} u_{1}+a_{2} u_{2}+a_{3} u_{12}, \quad B=b_{0}+b_{1} u_{1}+b_{2} u_{2}+b_{3} u_{12} \in A G(2)
$$

se tiene

$$
A B=c_{0}+c_{1} u_{1}+c_{2} u_{2}+c_{3} u_{12} \in A G(2)
$$

donde, usando la tabla del producto geométrico (3.3),

$$
\begin{aligned}
& c_{0}=a_{0} b_{0}+a_{1} b_{1}+a_{2} b_{2}-a_{3} b_{3} \\
& c_{1}=a_{0} b_{1}+a_{1} b_{0}+a_{3} b_{2}-a_{2} b_{3} \\
& c_{2}=a_{0} b_{2}+a_{2} b_{0}+a_{1} b_{3}-a_{3} b_{1} \\
& c_{3}=a_{0} b_{3}+a_{3} b_{0}+a_{1} b_{2}-a_{2} b_{1} .
\end{aligned}
$$

De otro lado,

- Identificando $u_{1}$ y $u_{2}$ con la base canónica de $\mathbb{R}^{2}$, explicitamos que AG(2) contiene una copia linealmente isomorfa de $\mathbb{R}^{2}$.

- Como $\left(u_{12}\right)\left(u_{12}\right)=-1$, usando la identificación $u_{12} \equiv \imath \in \mathbb{C}$, explicitamos que AG(2) contiene una copia linealmente isomorfa de los números complejos $\mathbb{C}$ que preserva la estructura de álgebra.

- Los resultados anteriores determinan el isomorfismo, como $\mathbb{R}$-espacios vectoriales:

$$
A G(2) \equiv \mathbb{C} \oplus \mathbb{R}^{2}
$$

En este nuevo contexto, usando la tabla del producto geométrico (3.3), el lector puede verificar los dos resultados algebraico-geométricos siguientes:

- En el Álgebra Geométrica AG(2) se cumple la Identidad Geométrica de Euler:

El producto geométrico de dos vectores no nulos, $u, v \in \mathbb{R}^{2}$, es el número complejo

$$
u v=\|u\|\|v\| \mathfrak{e}^{\theta \imath}=\|u\|\|v\| \cos \theta+\|u\|\|v\|(\sin \theta) \imath
$$

cuyo módulo es $|u v|=\|u\|\|v\|$ y cuyo argumento $\theta=\mu \varangle(u, v) \in]-\pi, \pi]$ es la medida del ángulo orientado de $u$ a $v$ (se conviene considerar $\theta=\pi$ si y solo si $u$ y $v$ son opuestos). Reciprocamente, todo número complejo es el producto geométrico de dos vectores, pero sin unicidad de los mismos.

Notar que, como $-\theta \in]-\pi, \pi]$ es la medida del ángulo orientado de $v$ a $u$, resulta que $v u$ es el complejo conjugado de $u v$ dado en (3.7), precisamente:

$$
v u=\|u\|\|v\| \mathfrak{e}^{-\theta \imath}=\|u\|\|\mid v\| \cos \theta-\|u\|\|v\|(\sin \theta) \imath
$$

- Los productos geométricos, $w z$ y $z w$, de un vector $w$ con un complejo $z$, son vectores simétricos respecto de $w$ que resultan de rotar el vector $|z| w$ (una homotecia de $w$ ) el ángulo orientado $\pm \arg (\mathrm{z})$ respectiva- 
mente, como sugiere el siguiente diagrama (con $|z|>1$ y $\left.0<\arg (z)<\frac{\pi}{2}\right)$ :

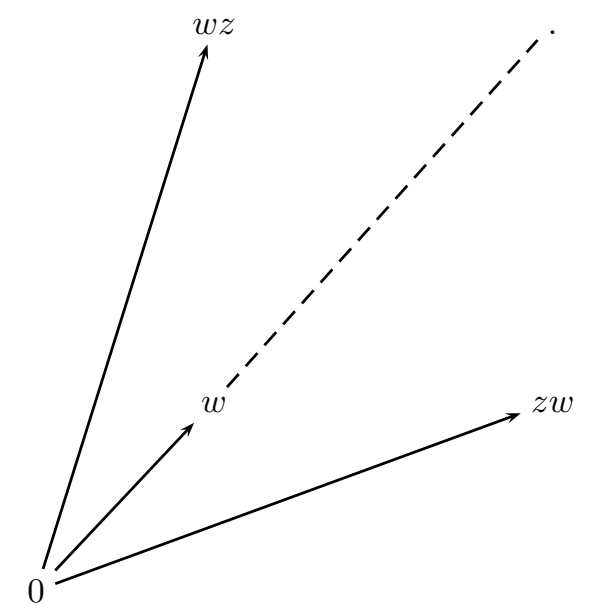

Productos geometricos de vector con complejo

\section{Algebrizando la Geometría: Algunos Ejemplos.}

PRIMERO: La estructura de álgebra geométrica permite determinar la conocida métrica euclideana en $\mathbb{R}^{2}$, que inicialmente hemos considerado simplemente como un espacio vectorial; en efecto, de la Identidad Geométrica de Euler (3.7) se tiene:

$$
\|v\|=\sqrt{v v} .
$$

SEGUNDO: La Identidad Geométrica de Euler (3.7) (y también (3.8)) algebriza de modo invariante (sin usar coordenadas) los conceptos geométricos de ortogonalidad y colinealidad de vectores: Dados los vectores no nulos $u \mathrm{y} v$

- $u v+v u=\|u\|\|v\| \cos \theta$, reestablece el producto interior $u . v$ lo que permite algebrizar de modo invariante la ortogonalidad de dichos vectores; en efecto,

$$
u \perp v \Longleftrightarrow u v+v u=0 \text { (su producto geométrico anticonmuta). }
$$

- $|u v-v u|=|| u|||| v|||\sin \theta|$, reestablece el valor del área del paralelogramo que determinan los vectores $u$ y $v$ lo que permite algebrizar de modo invariante la colinealidad de tales vectores; en efecto,

$$
u \| v \Longleftrightarrow u v-v u=0 \text { (su producto geométrico conmuta). }
$$

TERCERO: Las Condiciones de Dirac (3.4) corresponden a la versión algebraica del concepto de base ortonormal; en efecto, basta ver que la forma explícita de las Condiciones de Dirac son las conocidas Condiciones de Grassmann-Clifford:

$$
u_{1} u_{1}=u_{2} u_{2}=1 \quad \text { y } \quad u_{2} u_{1}=-u_{1} u_{2},
$$

y podemos concluir que las identidades (4.2) y (4.4) dicen: Los vectores $u_{1}$ y $u_{2}$ determinan una base base ortonormal si y solo si cumplen las Condiciones de Dirac.

CUARTO: Como $\arg (\imath)=\frac{\pi}{2}$, dado un vector unitario $u \in \mathbb{R}^{2} \backslash\{0\}$ podemos construir bases ortonormales orientadas: $(u, u \imath)$ positivamente orientada y $(u, \imath u)$ negativamente orientada; en efecto, es un caso particular del resultado graficado en (3.9) ya que los vectores $u \imath$ y $\imath u$ son ortogonales a $u$, de la misma logitud de $u$ y uno inverso del otro, precisamente:

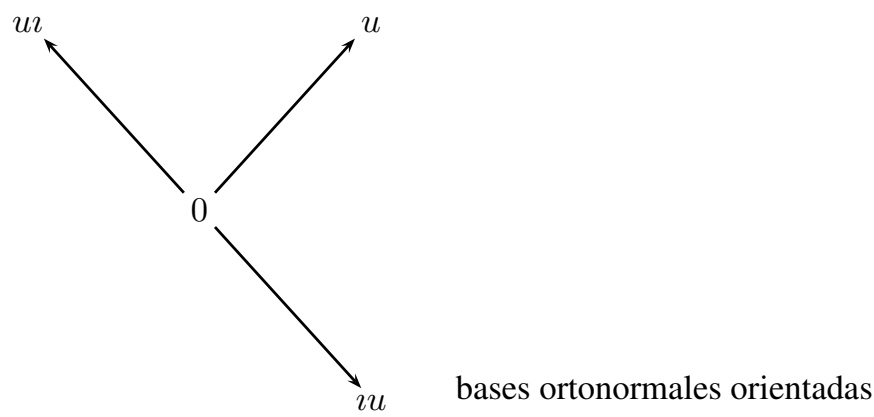


QUINTO: Podemos algebrizar el proceso geométrico de calcular el vector reflexión, $r_{u}(w) \in \mathbb{R}^{2}$ de un vector $w \in \mathbb{R}^{2}$, respecto a una recta $l$ que pasa por el origen y es ortogonal al vector unitario $u \in S^{1}$, mediante la siguiente aplicación, construida utilizando el producto geométrico de $\mathrm{AG}(2)$,

$$
\rho_{u}: v \in \mathbb{R}^{2} \longmapsto \rho_{u}(v)=-u v u \in \mathbb{R}^{2} .
$$

En efecto, el diagrama dado abajo nos ayuda a ver que

$$
w=(w \cdot u) u+(w \cdot(u \imath)) u \imath \Longrightarrow r_{u}(w)=-(w \cdot u) u+(w \cdot(u \imath)) u \imath
$$

De otro lado, calculando en el contexto de $\mathrm{AG}(2)$ y usando $-\imath u=u \imath, u u=1$, tenemos

$$
\rho_{u}(v)=-u w u=-u((w \cdot u) u+(w \cdot(u \imath)) u \imath) u=-(w \cdot u) u+(w \cdot(u \imath)) u \imath
$$

notar que $w . u$ y $w .(u \imath)$ son escalares y conmutan con todo número geométrico.

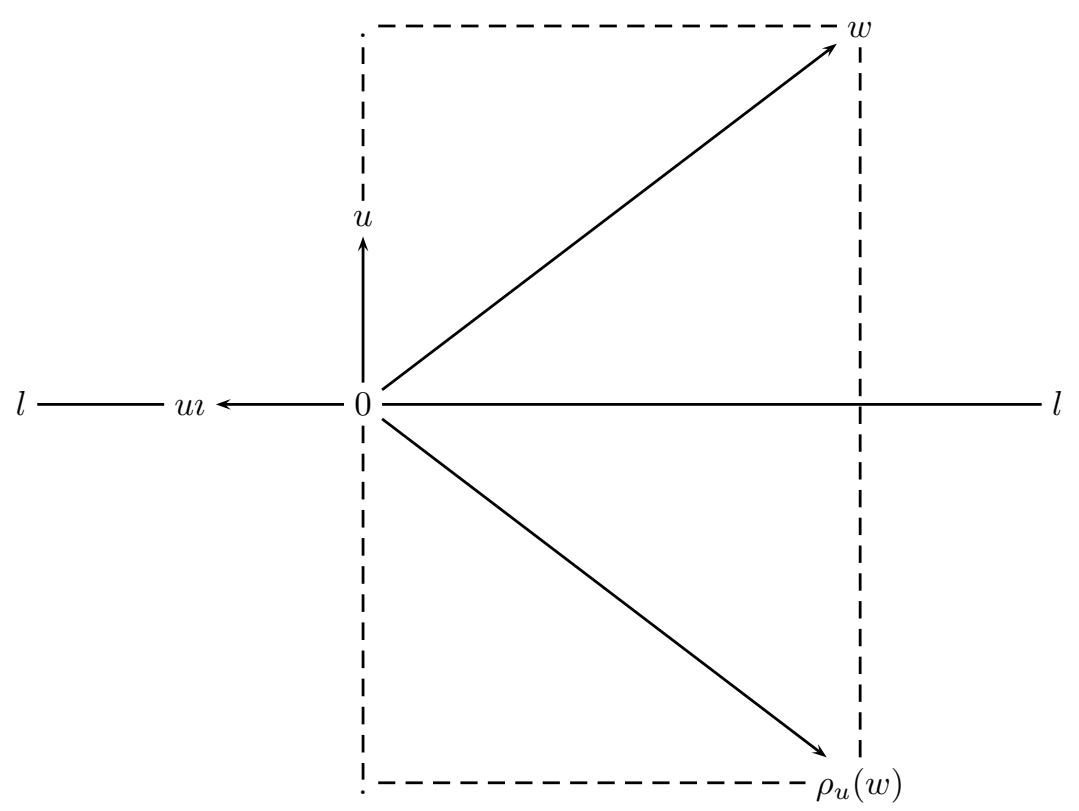

SEXTO: Usaremos el Álgebra Geométrica Afin para dar una demostración algebraica del siguiente resultado que, a rigor, corresponde a la Geometría Euclideana Afin:

En todo polígono convexo de $n \geq 3$ lados la suma de las medidas de sus ángulos interiores, orientados positivamente, es igual a $\pi(n-2)$.

Denotando con $A_{j} \in \mathbb{E}^{2}, \quad 1 \leq j \leq n$, los vértices del polígono convexo (considerados secuencialmente de tal modo que recorremos el polígono en el sentido antihorario) y con $\alpha_{j}$ el ángulo interior, positivamente orientado, correspondiente al vértice $A_{j}$, lo que debemos demostrar es:

$$
\sum_{j=1}^{n} \alpha_{j}=\pi(n-2)
$$

Este tipo de aplicación requiere unificar previamente lo hecho en las dos secciones anteriores y presentar resultados que nos permita extender el Álgebra Geométrica AG(2), del Espacio Vectorial Euclideano $\mathbb{R}^{2}$, al Álgebra Geométrica Afin AGA(2) del Espacio Afin Euclideano $\mathbb{E}^{2}$; por ejemplo,

- Ampliar el producto geométrico de vectores libres mediante

$$
(P X)(Q Y)=(\underline{P X})(\underline{Q Y})
$$

- Ampliar la Identidad Geométrica de Euler (3.7), a la Identidad Geométrica de Euler Afin: Dados los vectores libres no nulos $A B \in T_{A} \mathbb{E}^{2}$ y $X Y \in T_{X} \mathbb{E}^{2}$ se cumple:

$$
(A B)(X Y)=\|A B\|\|X Y\| \mathfrak{e}^{\theta \imath}
$$

donde $\theta=\mu \varangle(\underline{A B}, \underline{X Y}) \in]-\pi, \pi]$ (ángulo orientado) 
Aquí nos conviene reescribir la identidad Geométrica de Euler Afin (4.7) del siguiente modo: Dados los vectores libres no nulos $A B \in T_{A} \mathbb{E}^{2}$ y $X Y \in T_{X} \mathbb{E}^{2}$ se cumple:

$$
\frac{(A B)(X Y)}{\|A B|\||| X Y\|}=\mathfrak{e}^{\theta \imath}
$$

Veamos ahora la demostración de (4.6) para el caso de un triángulo:

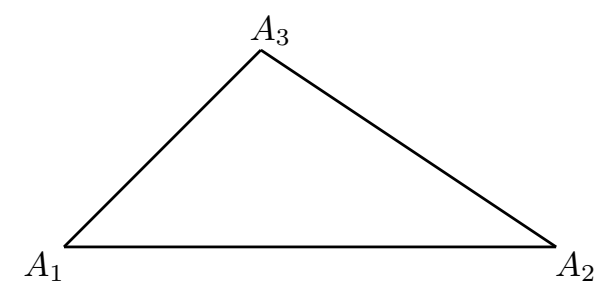

Con

$$
\alpha_{1}=\mu \varangle\left(\underline{A_{1} A_{2}}, \underline{A_{1} A_{3}}\right), \alpha_{2}=\mu \varangle\left(\underline{A_{2} A_{3}}, \underline{A_{2} A_{1}}\right) \text { y } \alpha_{3}=\mu \varangle\left(\underline{A_{3} A_{1}}, \underline{A_{3} A_{2}}\right) .
$$

Aplicando tres veces la identidad (4.8), resulta:

$$
\begin{aligned}
\mathfrak{e}^{\left(\alpha_{1}+\alpha_{2}+\alpha_{3}\right) \imath} & =\mathfrak{e}^{\alpha_{1} \imath \mathfrak{e}^{\alpha_{2} \imath} \mathfrak{e}^{\alpha_{3} \imath}} \\
& =\frac{\left(A_{1} A_{2}\right)\left(A_{1} A_{3}\right)}{\left\|A_{1} A_{2}\right\|\left\|A_{1} A_{3}\right\|} \frac{\left(A_{2} A_{3}\right)\left(A_{2} A_{1}\right)}{\left\|A_{2} A_{3}\right\|\left\|A_{2} A_{1}\right\|} \frac{\left(A_{3} A_{1}\right)\left(A_{3} A_{2}\right)}{\left\|A_{3} A_{1}\right\|\left\|A_{3} A_{2}\right\|} \\
& =\frac{\left(A_{2} A_{3}\right)\left(A_{2} A_{1}\right)}{\left\|A_{2} A_{3}\right\|\left\|A_{2} A_{1}\right\|} \frac{\left(A_{1} A_{2}\right)\left(A_{1} A_{3}\right)}{\left\|A_{1} A_{2}\right\|\left\|A_{1} A_{3}\right\|} \frac{\left(A_{3} A_{1}\right)\left(A_{3} A_{2}\right)}{\left\|A_{3} A_{1}\right\|\left\|A_{3} A_{2}\right\|} \\
& =-1
\end{aligned}
$$

Para simplificar hemos usado la anticonmutatividad $Y X=-X Y$ y, de la Identidad de Euler (4.7), $(X Y)(X Y)=$ $\|X Y\|^{2}$.

Podemos entonces concluir que para el caso de un triángulo; es decir, $\mathrm{n}=3$,

$$
\mathfrak{e}^{\left(\alpha_{1}+\alpha_{2}+\alpha_{3}\right) \imath}=-1 \Longrightarrow \alpha_{1}+\alpha_{2}+\alpha_{3}=\pi=(3-2) \pi
$$

Para el caso $n>3$ usaremos el proceso de inducción:

Considerando el segmento que une los vértices $A_{2}$ y $A_{n}$ tendremos un triángulo T, que une los vértices $A_{1}, A_{2}$ y $A_{3}$ y un polígono convexo $\mathrm{P}$ de $\mathrm{n}-1$ lados, que une los vértices de $A_{2}, A_{3} \ldots A_{n}$. Hecho esto, aplicando inducción tendremos que la suma de las medidas de los ángulos interiores del polígono convexo de $\mathrm{n}$ lados, $\pi(n-2)$, resulta de sumar $\pi(n-1-2)$ (la suma de las medidas de los ángulos interiores del polígono convexo de n-1 lados P) con $\pi$ (la suma de las medidas de los ángulos interiores del triángulo T).

5. Conclusiones. Permiten explicar el nombre Álgebra Geométrica, dado por William K. Clifford, quién fué su creador en el periodo comprendido entre1873 y 1879.

- La estructura afin del espacio euclideano bidimensional nos ha permitido, por ejemplo, matematizar la suma de vectores libres (2.1) mediante la suma de vectores en $\mathbb{R}^{2}$.

- El Álgebra Geométrica AG(2) amplía y optimiza la relación álgebra-geometría y torna mucho más amigable el conocimiento y uso de los complejos. Nos ha permitido, por ejemplo, presentar la Identidad Geométrica de Euler (3.7), llamada así porque, considerando $u$ y $v$ unitarios se obtiene la conocida identidad de Euler

$$
\mathfrak{e}^{\theta \imath}=\cos \theta+(\sin \theta) \imath \quad ; \quad \theta \in \mathbb{R},
$$

cuyo caso particular ( $u$ unitario y $v=-u$, en cuyo caso $\theta=\mu \varangle(u,-u)=\pi$ ) es la famosa Ecuación de Euler, conocida desde antes de 1748,

$$
\mathfrak{e}^{\pi \imath}+1=0
$$

- El producto geométrico de AG(2) permite algebrizar no solo el concepto geométrico de ortogonalidad (ver (4.2)), sino también los conceptos de paralelismo (ver (4.3)), base ortonormal (ver el Ejemplo TERCERO) y las reflexiones en $\mathbb{R}^{2}$ (ver el Ejemplo QUINTO). 
En este proceso se redefine el producto interior de vectores: $u \cdot v=\frac{1}{2}(u v+v u)$, que algebriza la ortogonalidad. Se introduce el producto exterior de los mismos: $u \wedge v=\frac{1}{2}(u v+v u)$, que algebriza el paralelismo y finalmente se obtiene la relación unificadora $u v=u . v+u \wedge v$.

Agradecimientos. Al Comité Editor de SELECCIONES MATEMÁTICAS por su amable acogida, en particular, al Dr. Obidio Rubio por su gentil apoyo.

Referencias

[1] Artin E., Algebra Geométrica; Limusa, Méjico 1992.

[2] Berger M., Geometry I; Springer, Heildelberg, 1987.

[3] Barker - Howe Continuous Symmetry (From Euclid to Klein); American Mathematical Society, 2007.

[4] Vera E. Matemática Básica para Docentes Universitários de Matemática; Notas de Clase, 2017. 